\title{
Petrophysics and Fluid Transport in Shales and Tight Reservoirs
}

\author{
Feng Yang $\mathbb{D},{ }^{1}$ Xiaohu Dong, ${ }^{2}$ Alexandra Amann-Hildenbrand, ${ }^{3}$ Ebrahim Fathi, ${ }^{4}$ \\ and Derek Elsworth ${ }^{5}$ \\ ${ }^{1}$ China University of Geosciences, Wuhan, China \\ ${ }^{2}$ China University of Petroleum, Beijing, China \\ ${ }^{3}$ RWTH Aachen University, Aachen, Germany \\ ${ }^{4}$ West Virginia University, Morgantown, WV, USA \\ ${ }^{5}$ Pennsylvania State University, University Park, PA, USA \\ Correspondence should be addressed to Feng Yang; fengyang@cug.edu.cn
}

Received 21 May 2018; Accepted 23 May 2018; Published 5 August 2018

Copyright (c) 2018 Feng Yang et al. This is an open access article distributed under the Creative Commons Attribution License, which permits unrestricted use, distribution, and reproduction in any medium, provided the original work is properly cited.

\section{Motivation and Background}

The development of horizontal drilling and hydraulic fracturing has enabled the extraction of hydrocarbons from fine-grained sedimentary rocks. This type of unconventional resource has gained much attention for most oil companies all over the world. Understanding petrophysics and the gas-water-rock interactions is essential in guaranteeing the effective recovery of hydrocarbons from shale and other tight reservoirs. But shales and tight rocks are commonly highly heterogeneous with behaviors tiered in multiple scales $[1,2]$. Also, within the reservoir, the coexistence of micro-/ nanopores and natural/hydraulic fractures further enhances the complexity of storage and transport spaces $[3,4]$. During the last decade, the development of effective experimental and numerical methods to explore the petrophysics in shale and tight reservoirs has become a top priority for the exploitation of shale and tight reservoirs.

Fluid transport in such complex media cannot be easily determined with conventional techniques, which are successfully applied for reservoir rocks. At micro-/nanoscales, the interplay between fluids and the pore wall is no longer negligible. The mechanisms of diffusion, slip flow, and sorption all significantly affect the transport of geofluids $[5,6]$. Moreover, such small diameter storage and transport pores/fractures enhance the effect of pore confinement on the phase behavior of fluids $[7,8]$. The bubble point pressure and phase envelop of reservoir fluids are changed. The focus of this special issue is therefore placed on an improved understanding of the phase behavior of geofluids, fluid transport mechanisms, and non-Darcy effects in nanopores of shales and other tight rocks. The purpose of this special issue is to collate high-quality research articles in petrophysics and fluid transport in unconventional reservoirs. The special issue addresses the most recent advances in physical experiments and numerical simulation techniques to study the petrophysics and gas-water-rock interactions of unconventional reservoirs across broad length and time scales.

\section{Contents of the Special Issue}

2.1. Dielectric Properties of Fine-Grained Sedimentary Rocks. As contemporary petroleum exploration activities have focused on unconventional resources, the traditional experimental methods and numerical simulation tools are sometimes no longer effective. We confront numerous challenges to accurately describe the petrophysics of fine-grained sedimentary rocks in unconventional tight and shale reservoirs.

R. Beloborodov et al. experimentally characterize the dielectric properties of fluid-saturated artificial shales. 
Because of the high dielectric contrast between water and hydrocarbons, the producible layers of reservoir rocks and surrounding media can be effectively distinguished. In this paper, the authors investigate the frequency-dependent dielectric properties of artificial shale rocks prepared from silt-clay mixtures via mechanical compaction.

T. Han et al. present a theoretical model for the anisotropic dielectric properties of artificial shales. Their model is based on the theoretical assumption of differential effective medium models for any number of mineral grain components aligned in any directions and is shown to be independent of the mixing order. By incorporating a measured orientation distribution function of the clay particles and by inverting the dielectric properties of the artificial sample composed of clay and brine, their model is capable of modeling the frequency-dependent anisotropic dielectric properties of artificial shales.

2.2. Fluid Transport in Shales and Tight Reservoirs. Gas shales and tight reservoirs exhibit extremely low permeability. This low permeability makes it difficult to apply traditional transport modeling approaches, such as Darcy's law, to shales $[9,10]$. Gas flow in organic matter, natural fractures, and hydraulic fractures occurs via different mechanisms: continuum flow, slip flow, and Knudsen diffusion. Adsorbed gas desorption from the organic matter also plays a role in gas transport [11]. It is challenging to develop a comprehensive model to describe the multiple mechanisms in the gas transport process.

S. Huang et al. proposed a comprehensive apparent permeability model to consider the multiple transport mechanisms in shale gas reservoirs. The specific mechanisms include viscous flow, slip flow, Knudsen diffusion, and surface diffusion. In their model, the pore diameter and mean free path of gas molecules are corrected by considering the adsorption layer and dense gas effect.

Y. Zeng et al. develop a modified apparent permeability model to describe gas flow in shale gas reservoirs. The apparent model integrates viscous flow, Knudsen diffusion, and gas desorption. They additionally consider a macroseepage model of multistage fractured horizontal wells accommodating multiple gas flow mechanisms to predict the dynamic pressure and production performance.

Hydrocarbon extraction from tight reservoirs is feasible using multiple-fractured horizontal wells. The technology to create multiple-fractured horizontal wells creates a complex stimulated reservoir volume (SRV) with induced fractures proximal to the hydraulic fractures. J. Wang et al. focus on the geometric properties of this stimulated reservoir volume in tight reservoirs and develop a new semianalytical model to analyze the well bottom pressure response. The calculations are helpful to understand the dynamic characteristics of multiple-fractured horizontal wells and the performance of the stimulated reservoir volume.

Hydraulic fracturing is one of the key methods for the effective development of unconventional reservoirs. In the process of hydraulic fracturing, a significant volume of fracturing fluid is injected into the reservoir. However, much of this fracturing fluid is retained in the formation after flow-back [12]. Y. Shen et al. study the aqueous phase trapping phenomenon in volcanic reservoirs. Nuclear magnetic resonance (NMR) and pulse-decay permeability techniques are employed to monitor the spontaneous imbibition of the fracturing fluid. This work improves our understanding of transport mechanisms of hydraulic fracturing fluids in unconventional reservoirs.

\section{Acknowledgments}

We appreciate the significant contributions of the various authors to this special issue and their perseverance during the publication process. We also thank the many anonymous reviewers who helped evaluate and contribute to these papers.

Feng Yang
Xiaohu Dong
Alexandra Amann-Hildenbrand
Ebrahim Fathi
Derek Elsworth

\section{References}

[1] J. B. Curtis, "Fractured shale-gas systems," AAPG Bulletin, vol. 86, no. 11, pp. 1921-1938, 2002.

[2] F. Yang, Z. Ning, Q. Wang, R. Zhang, and B. M. Krooss, "Pore structure characteristics of lower Silurian shales in the southern Sichuan Basin, China: insights to pore development and gas storage mechanism," International Journal of Coal Geology, vol. 156, pp. 12-24, 2016.

[3] A. Amann-Hildenbrand, A. Ghanizadeh, and B. M. Krooss, "Transport properties of unconventional gas systems," Marine and Petroleum Geology, vol. 31, no. 1, pp. 90-99, 2012.

[4] Y. Gensterblum, A. Ghanizadeh, R. J. Cuss et al., "Gas transport and storage capacity in shale gas reservoirs - a review. Part A: transport processes," Journal of Unconventional Oil and Gas Resources, vol. 12, pp. 87-122, 2015.

[5] I. Y. Akkutlu and E. Fathi, "Multiscale gas transport in shales with local kerogen heterogeneities," SPE Journal, vol. 17, no. 4, pp. 1002-1011, 2012.

[6] E. Fathi and I. Y. Akkutlu, "Matrix heterogeneity effects on gas transport and adsorption in coalbed and shale gas reservoirs," Transport in Porous Media, vol. 80, no. 2, pp. 281-304, 2009.

[7] X. Dong, H. Liu, J. Hou, K. Wu, and Z. Chen, "Phase equilibria of confined fluids in nanopores of tight and shale rocks considering the effect of capillary pressure and adsorption film," Industrial \& Engineering Chemistry Research, vol. 55, no. 3, pp. 798-811, 2016.

[8] X. Dong, H. Liu, W. Guo, J. Hou, Z. Chen, and K. Wu, "Study of the confined behavior of hydrocarbons in organic nanopores by the potential theory," Fluid Phase Equilibria, vol. 429, pp. 214-226, 2016.

[9] K. Fan, M. Dong, D. Elsworth, Y. Li, C. Yin, and Y. Li, “A dynamic-pulse pseudo-pressure method to determine shale matrix permeability at representative reservoir conditions," International Journal of Coal Geology, vol. 193, pp. 61-72, 2018.

[10] X. Cui, A. M. M. Bustin, and R. M. Bustin, "Measurements of gas permeability and diffusivity of tight reservoir rocks: 
different approaches and their applications," Geofluids, vol. 9, no. 3, 223 pages, 2009.

[11] K. Wu, X. Li, C. Wang, W. Yu, and Z. Chen, "Model for surface diffusion of adsorbed gas in nanopores of shale gas reservoirs," Industrial \& Engineering Chemistry Research, vol. 54, no. 12, pp. 3225-3236, 2015.

[12] J. Wang, D. Elsworth, and M. K. Denison, "Hydraulic fracturing with leakoff in a pressure-sensitive dual porosity medium," International Journal of Rock Mechanics and Mining Sciences, vol. 107, pp. 55-68, 2018. 

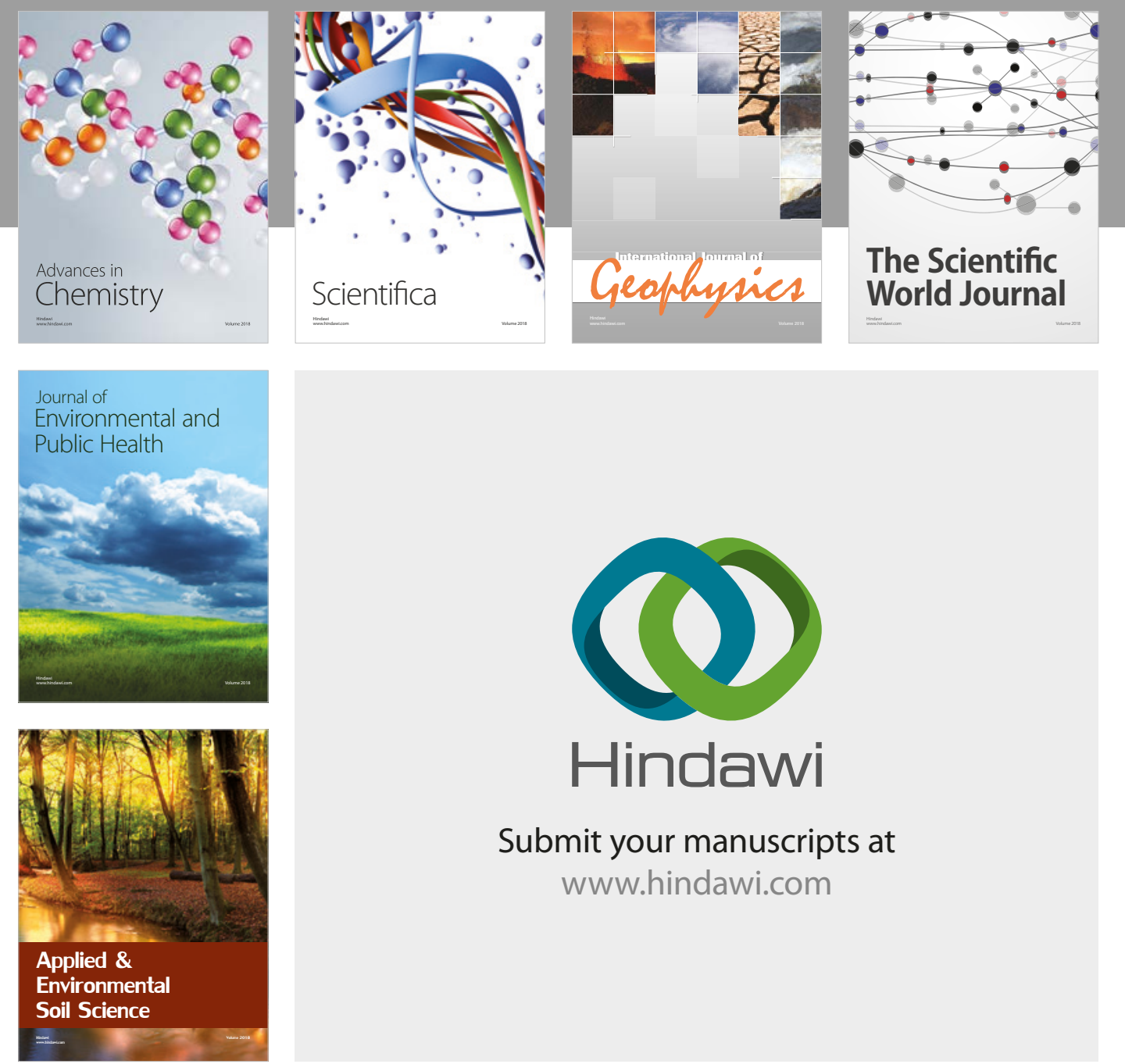

The Scientific

\section{World Journal}
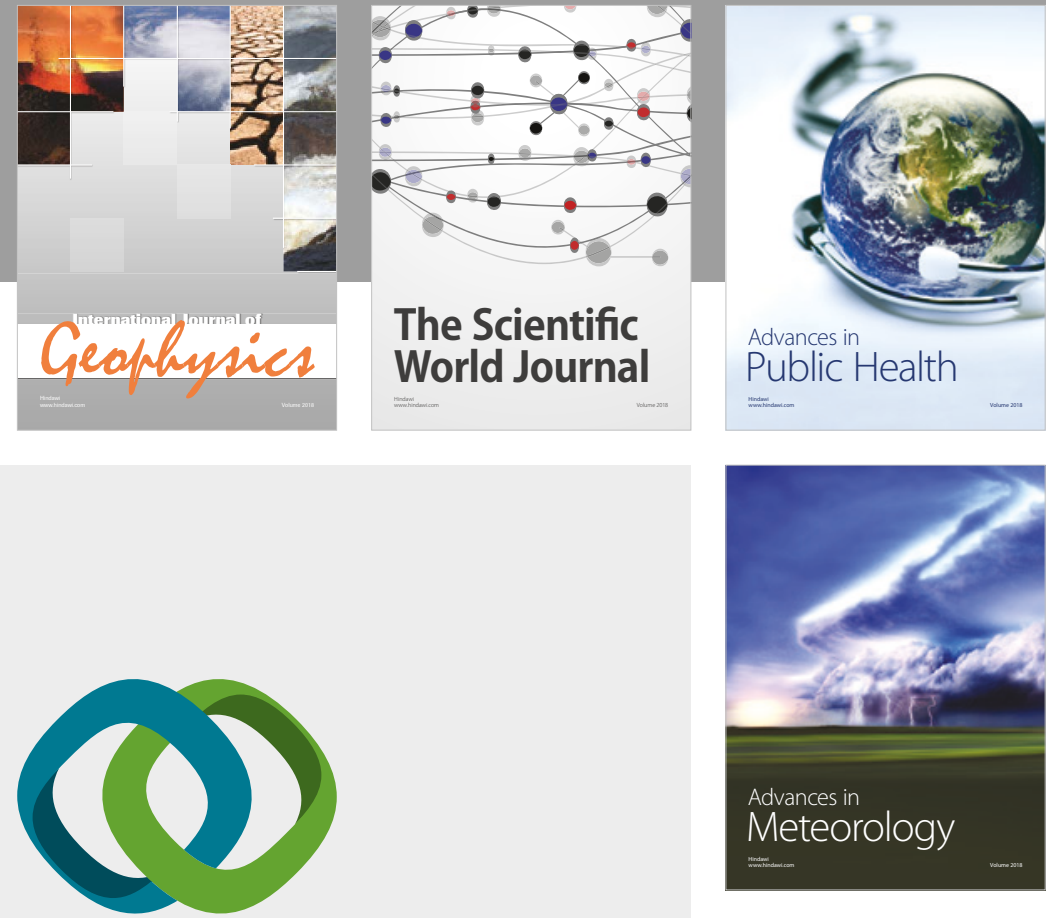

Advan

Public Health

\section{Hindawi}

Submit your manuscripts at

www.hindawi.com
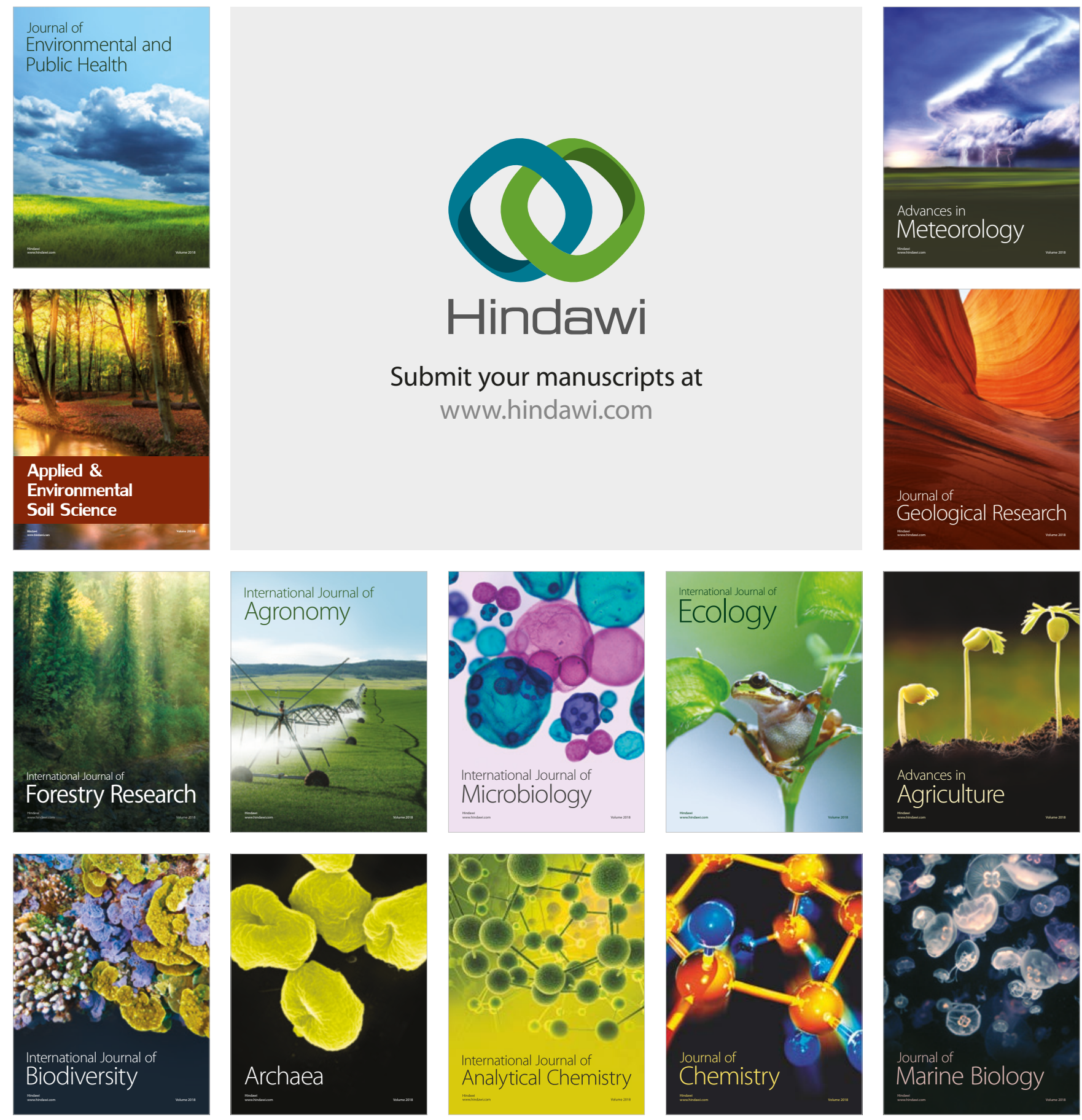